\title{
Analysis of CT Image Denoising Techniques
}

\author{
Anam Kazi ${ }^{1}$, Roshni Padate ${ }^{2}$ \\ Student, Electronics, Fr. C.R.C.E., Mumbai, India ${ }^{1}$ \\ Assistant Professor, Computer Science, Fr. C.R.C.E., Mumbai, India ${ }^{2}$
}

\begin{abstract}
Effective technique for denoising is necessary for medical images particularly in Computed Tomography, which is a significant and most general modality in medical imaging. In this work denoising of Computed Tomography (CT) images is performed which generally gets degraded by the presence of Additive White Gaussian Noise (AWGN). This work presents three techniques to denoise CT images namely Discrete Wavelet Transform (DWT) Thresholding, Non-local (NL) means filter and wavelet thresholding and joint NL means filtering. In this report, we explore wavelet denoising of images using several thresholding techniques such as SURE Shrink, Visu Shrink, Bayes Shrink and Neigh Shrink. NL-means filter assumes that the image contains an extensive amount of redundancy and therefore it uses the concept of self-similarity in an image to denoise it. The third technique is a combination of the above two methods. Data evaluations are accomplished by using two criterions; namely, peak signal to noise ratio (PSNR) and mean square error (MSE). It is observed that NL means performs better Wavelet denoising.
\end{abstract}

Keywords: CT image denoising, AWGN, Wavelet Thresholding, NL means, PSNR, Computation time.

\section{INTRODUCTION}

Digital images play a very important part in applications such as television magnetic resonance imaging, computer tomography as well as in field of science and technology such as geographical information system and astronomy. Sets of data collected by image sensors and other devices are generally contaminated by noise. Also noise gets introduced due to transmission errors and compression. Hence denoising is often a necessary and first step to be performed before image data is analyzed and processed. An efficient denoising technique must be applied to compensate for such data corruption. Image de-noising is an important pre-processing step for image analysis. It refers to the task of recovering a good estimate of the true image from a degraded observation without altering and changing useful structure in the image such as discontinuities and edges. Image denoising still remains an important challenge for researchers because denoising process removes noise but introduces artifacts and also causes blurring.

With the widespread use of digital imaging in medicine, the quality of digital medical images has become an important issue today. To achieve the best possible diagnoses, it is important for medical images to be sharp, clear, and free of noise and artifacts [1]. Though, the technologies for acquiring digital medical images continue to improve, resulting in images of higher and higher resolution and quality, noise remains as an issue in many medical images applications [2]. Removing noise in these digital images remains as one of the major challenges in the study of medical imaging [3].

\section{A.W.G.N.}

Additive white Gaussian noise, salt pepper noise and speckle noise are the types of noise generally found in images. The modifiers denote specific characteristics additive specifies noise gets added to each pixel intensity; white specifies that the spectral density of the noise is constant regardless of frequency and Gaussian term specifies that the noise has its Probability density function (PDF) equal to that of the normal distribution which is also known as the Gaussian amplitude distribution. Among various image-denoising strategies, the transform-domain approaches in general, and in particular the multiscale ones, are very efficient for AWGN reduction [4]. The AWGN has to be reduced through processing without affecting important features of the image.

\section{III.NEED FOR CT IMAGE DENOISING}

Considering the growth of the population undergoing CT scans and their exposure to the radiation, the public health risk may be significant. One researcher claimed that a population as big as $0.4 \%$ of the United States patients diagnosed by cancer, may be attributable to the radiation from CT studiesbased on CT usage data from 1991-1996 [5]. It was determined that $1.5-2 \%$ of cancers may eventually be resulted from the ionization radiation in CT scanning, when organ specific cancer risk was adjusted for current levels of CT usage [5]. Based on the mentioned study and similar ones the CT community was forced to review the prescribed amount of radiation for CT scans, especially for pediatric patients. Eventually this progress resulted in an effort to minimize CT doses and optimize image quality. 


\section{IV.WAVELET THRESHOLDING}

Wavelet denoising attempts to remove the noise present in the signal while preserving the signal characteristics, regardless of its frequency content [6]. The wavelet tresholding first proposed by Donoho removes noise by killing coefficients that are insignificant relative to some threshold, and turns out to be simple and effective.

A.Denoising Procedure

The procedure to denoise an image is given as follows:

De-noised image $=\mathrm{W}^{-1}[\mathrm{~T}\{\mathrm{~W}($ Original Image + Noise $)\}]$

Step 1: Apply forward wavelet transform to a noisy image to get decomposed image.

Step 2: Apply non-linear tresholding to decomposed image to remove noise.

Step 3: Apply inverse wavelet transform to thresholded image to get a denoised image in spatial domain.

B. Discrete Wavelet Transform

In DWT the original image is transformed into four sub bands, which is normally labeled as LL1, LH1, HL1 and HH1. The LL1 sub band comes from low pass filtering approximation. The remaining sub bands are called detailed sub bands. The HL1 comes from low pass filtering in the vertical direction and high pass filtering in the horizontal direction and so has the label HL1, it is also known as the horizontal fluctuation. The LH1 sub band comes from high pass filtering in the vertical direction and low pass filtering in the horizontal direction so it is labeled by LH1, it is also known as the vertical fluctuation and the HH1 sub band comes from high pass filtering in both direction so it is Diagonal Fluctuation labeled by HH1. To obtain the next level of decomposition, sub band LL1 alone is further decomposed into four sub bands labeled as LL2, LH2, HL2 and HH2. This process continues until some final scale is reached. After $\mathrm{L}$ decompositions, a total of $\mathrm{D}(\mathrm{L})=3 * \mathrm{~L}+1$ sub bands are obtained.

\section{Threshold Selection Rules}

Visu Shrink (VS) [6]:Visu Shrink is tresholding by applying the universal threshold proposed by Donoho and Johnstone [7][8]. This threshold is given by

$\lambda=\sigma \cdot 2 \log n^{2}$

where $\sigma$ is the noise level and $\mathrm{n}$ is the length of the noisy image. A highly smoothed estimate is obtained by Visu shrink since a common threshold is applied to all sub bands with less adaptability.

Normal Shrink (NS) [8]:The threshold is computed using

$$
\lambda=\beta \sigma^{2} / \sigma_{\mathrm{y}}
$$

$$
\begin{gathered}
\beta=\sqrt{\log \left(\frac{\mathrm{J}_{\mathrm{k}}}{\mathrm{L}}\right)} \\
\sigma_{\text {noise }}^{2}=\left[\frac{\operatorname{median}|\mathrm{y}(\mathrm{i}, \mathrm{j})|]^{2}}{0.675}\right]^{2} \\
\sigma_{\mathrm{y}}^{2}=\frac{1}{\mathrm{~N}_{\mathrm{s}}} \sum_{\mathrm{x}, \mathrm{y}}^{\mathrm{N}_{\mathrm{s}}} \mathrm{G}_{\mathrm{x}, \mathrm{y}}
\end{gathered}
$$

where $\beta$ is the scale parameter and is computed once for every scale, $\sigma_{\mathrm{y}}$ is the standard deviation of the sub band under consideration, $\sigma_{\text {noise }}$ is the noise variance which is estimated from the sub band HH1 where $\mathrm{J}_{\mathrm{k}}$ is the length of the subband at kth scale, $\mathrm{L}$ is the number of decomposition level, $\mathrm{y}(\mathrm{i}, \mathrm{j})$ are $\mathrm{HH}$ sub band coefficients.

Bayes Shrink (BS) [9]: The threshold is computed using

$$
\begin{gathered}
\lambda_{\beta}=\frac{\hat{\sigma}_{\text {noise }}}{\sqrt{\max \left(\left(\mathrm{o}_{\mathrm{G}}^{2}-\sigma_{\text {noise }}^{2}, 0\right)\right.}} \\
\sigma_{\mathrm{y}}^{2}=\frac{1}{\mathrm{~N}_{\mathrm{s}}} \sum_{\mathrm{x}, \mathrm{y}}^{\mathrm{N}_{\mathrm{s}}} \mathrm{G}_{\mathrm{x}, \mathrm{y}}{ }^{2} \\
\sigma_{\text {noise }}^{2}=\left[\frac{\operatorname{median}|\mathrm{y}(\mathrm{i}, \mathrm{j})|}{0.675}\right]^{2}
\end{gathered}
$$

$\sigma_{\mathrm{y}}$ is the standard deviation of the sub band under consideration, $\sigma_{\text {noise }}$ is the noise variance which is estimated from the sub band $\mathrm{HH} 1, \mathrm{G}_{\mathrm{x}, \mathrm{y}}$ is the coefficients of the $\mathrm{HH}$ subband, $\mathrm{N}_{\mathrm{s}}$ is length 


\section{NON LOCAL MEANS BASED DENOISING}

\section{A. Non-local Means Theory}

The non-local means algorithm assumes the image contains an extensive amount of self-similarity. An example of selfsimilarity is displayed in Figure 1 below. The figure shows three pixels $\mathrm{p}, \mathrm{q} 1$, and $\mathrm{q} 2$ and their respective neighbourhoods. The neighborhoods of pixels $\mathrm{p}$ and $\mathrm{q} 1$ are similar, but the neighborhoods of pixels $\mathrm{p}$ and $\mathrm{q} 2$ are not similar. Adjacent pixels tend to have similar neighborhoods, but non-adjacent pixels will also have similar neighborhoods when there is structure in the figure.

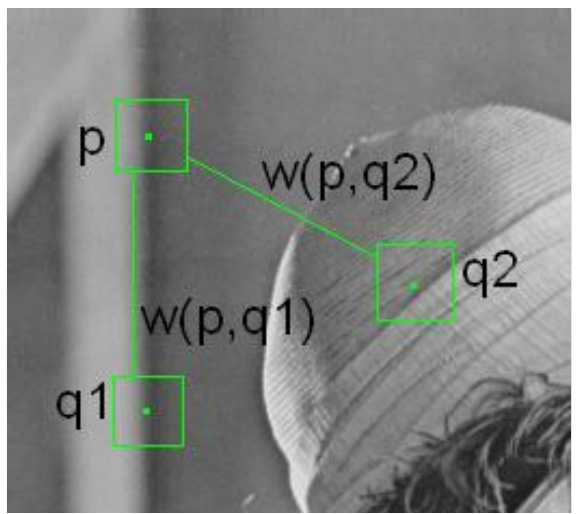

Fig 1 Pixel Neighborhoods

B. Non-local Means Method

Each pixel i of the non-local means denoised image is computed with the following formula:

$N L[v](i)=\sum_{j \in I} w(i, j) v(j)$

Where, $\mathrm{v}=\{\mathrm{v}(\mathrm{i}) \mid \mathrm{i} \in I\}$ is the noisy image, and weights $\mathrm{w}(\mathrm{i}, \mathrm{j})$ meet the following conditions $0<\mathrm{w}(\mathrm{i}, \mathrm{j})<1$ and $\sum_{j} w(i, j)=$ 1. Two pixels $i$ and $j$ are similar if the intensity gray level vectors $v(\mathrm{Ni})$ and $\mathrm{v}(\mathrm{Nj})$ are similar, where $\mathrm{Nk}$ denotes a square neighborhood of fixed size and centered at a pixel $\mathrm{k}$. This similarity is measured as a decreasing function of the weighted Euclidean distance [4] $\|\left(v\left(N_{i}\right)-v\left(N_{j}\right) \|_{2, a}^{2}\right.$, where a $>0$ is the standard deviation of Gaussian kernel. The Euclidean distance when applied to noisy neighborhoods raises the following equality [4],

$$
E\left\|v\left(N_{i}\right)-v\left(N_{j}\right)\right\|_{2, a}^{2}=\left\|u\left(N_{i}\right)-u\left(N_{j}\right)\right\|_{2, a}^{2}+2 \sigma^{2}
$$

The weights are defined as [4],

$$
w(i, j)=\frac{1}{Z(i)} e^{\left\|v\left(N_{i}\right)-v\left(N_{j}\right)\right\|_{2, a}^{2}}
$$

$\mathrm{Z}(\mathrm{i})$ is the normalizing constant defined as,

$$
Z(i)=\sum_{i} e^{-\frac{\left\|v\left(N_{i}\right)-v\left(N_{j}\right)\right\|_{2, a}^{2}}{h^{2}}}
$$

And the parameter $\mathrm{h}$ acts as a degree of filtering. It controls the dcay of the exponential function. The advantage of this method is that it preserves image details when denoisng.

\section{VI.WAVELET THRESHOLDING AND JOINT NL MEANS FILTERING}

In this proposed technique a combination of the above two techniques is used. The given noisy image has been subjected to two levels of wavelet decomposition and in each level, the detail subband coefficients are subjected to hard wavelet thresholding, where BayesShrink determines the threshold $\mathrm{T}$. While reconstructing the image from the wavelet coefficients by applying inverse wavelet transform, the level 1 approximation subband coefficients are subjected to NLM means filtering. The neighborhood size of given pixel is selected as $3 \times 3$. The search window size is selected as $7 \mathrm{X} 7$. The degree of averaging $\mathrm{h}$ is selected as the estimated noise std $\sigma_{\text {noise }}$. 


\section{IMAGE ASSESSMENT PARAMETERS}

Mean square Error (MSE)

$$
M S E=\frac{1}{N^{2}} \sum_{x, y=0}^{N-1} \sum\left(X_{x, y}-\hat{X}_{x, y}\right)^{2}
$$

Peak Signal to noise ratio (PSNR)

$$
\operatorname{PSNR}(d b)=10 \log _{10}\left(\frac{255 \times 255}{M S E}\right)
$$

\section{RESULTS AND DISCUSSIONS}

The algorithms are implemented using MATLAB R2013b tool. The test CT scan images required to implement the algorithm are obtained from www.ctisus.com by the John Hopkins Hospital. The images are of JPEG format. The algorithms are tested on images of different parts of the body like adrenal, cardiac, gastrointestinal, kidneys, pancreas, small bowls and spleen. The three techniques for AWGN noise removal are compared in this work; Wavelet Thresholding, Non Local (NL) means and wavelet thresholding and joint NL means based image denoising. Wavelet Thresholding technique is implemented by using the three thresholding rules namely Visu shrink, Normal Shrink and Bayes Shrink. Calculating the Mean Square Error (MSE) and Peak Signal to Noise Ratio (PSNR) does performance evaluation of the techniques.
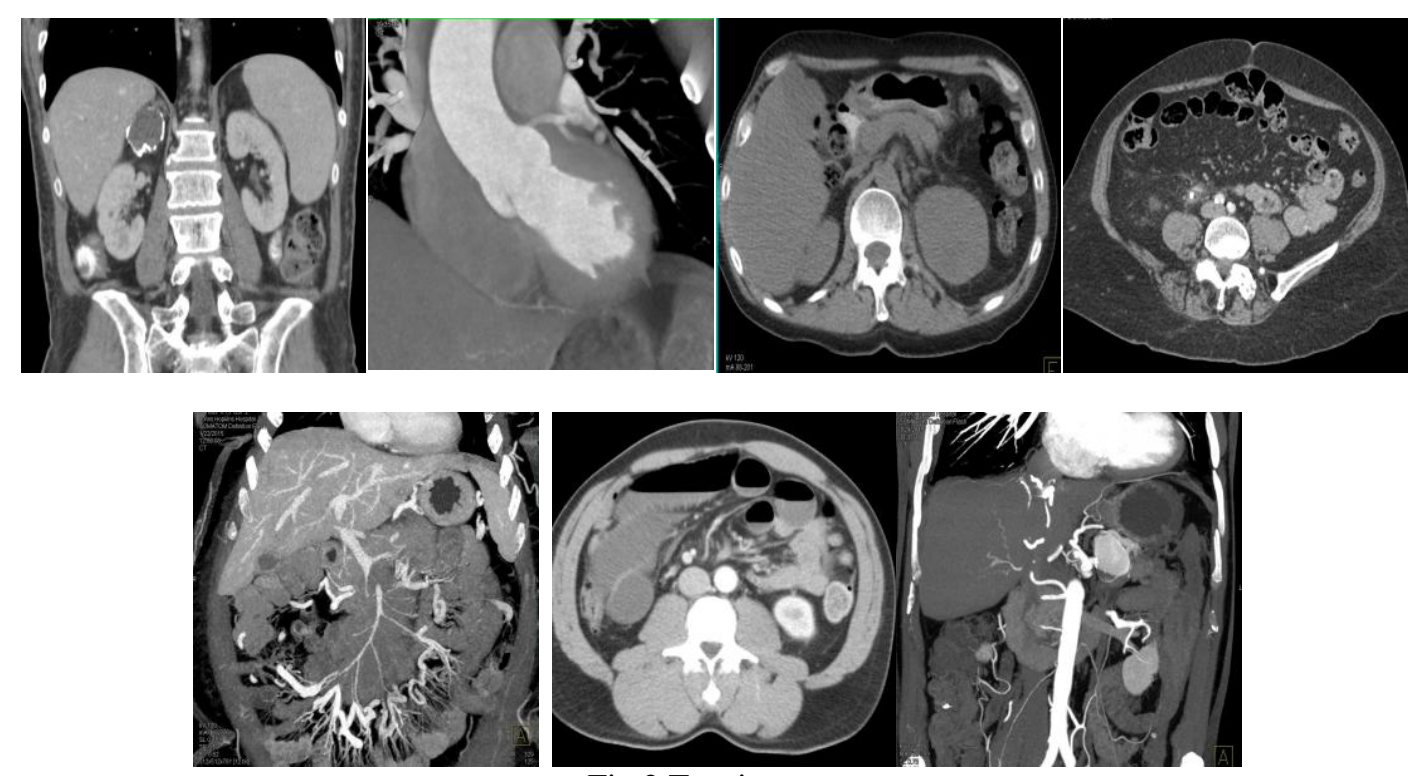

Fig 2 Test images.

(L-R) Adrenal, Cardiac, Gastrointestinal, Kidney, Pancreas, Small Bowl , Spleen

TABLE I PSNR VALUES (DB)

Test Images

Adrenal

Cardiac

\section{Wavelet Transform Thresholding}

Noise std. Sure Normal Bayesian deviation shrink shrink

$$
29.51
$$

23.22

19.82

29.57

23.19

19.63
NL means
Wavelet Thresholding and Joint NL Means Filtering

$$
30.3
$$


International Journal of Advanced Research in Computer and Communication Engineering ISO 3297:2007 Certified

Vol. 6, Issue 4, April 2017

Gastro intestinal

\begin{tabular}{|c|c|c|c|c|c|c|}
\hline & 10 & 28.44 & 20.25 & 31.77 & 29.3 & 31.82 \\
\hline & 20 & 22.91 & 23.72 & 25.33 & 23.22 & 25.44 \\
\hline & 30 & 19.51 & 20.25 & 21.6 & 19.83 & 21.52 \\
\hline \multicolumn{7}{|l|}{ Kidney } \\
\hline & 10 & 28.15 & 3.3 & 33.07 & 29.74 & 33.05 \\
\hline & 20 & 22.88 & 23.86 & 25.34 & 23.26 & 25.38 \\
\hline & 30 & 19.7 & 20.32 & 21.11 & 19.81 & 21.21 \\
\hline \multicolumn{7}{|l|}{ Pancreas } \\
\hline & 10 & 27.13 & 30.39 & 35.8 & 29.38 & 17.33 \\
\hline & 20 & 22.06 & 23.29 & 26.35 & 22.83 & 26.41 \\
\hline & 30 & 18.91 & 19.58 & 21.05 & 19.34 & 21.09 \\
\hline \multicolumn{7}{|l|}{ Small bowls } \\
\hline & 10 & 28.54 & 30.06 & 32.11 & 29.52 & 32.06 \\
\hline & 20 & 23.08 & 23.88 & 25.44 & 23.34 & 25.2 \\
\hline & 30 & 19.76 & 20.33 & 21.35 & 19.82 & 21.69 \\
\hline \multicolumn{7}{|l|}{ Spleen } \\
\hline & 10 & 27.57 & 31.16 & 35.63 & 29.16 & 35.67 \\
\hline & 20 & 22.26 & 23.9 & 27.37 & 25.03 & 27.29 \\
\hline & 30 & 19.12 & 20.13 & 22.28 & 19.57 & 22. \\
\hline
\end{tabular}

TABLE II COMPUTATION TIME

Computation Time (secs)

Test images

Noise std deviation

NL means

581.2

588.34

939.8

Cardiac

Gastro

intestinal

\begin{tabular}{|cc} 
& $\mathbf{1 0}$ \\
& $\mathbf{2 0}$ \\
Kidney & $\mathbf{3 0}$ \\
& $\mathbf{1 0}$ \\
& $\mathbf{2 0}$ \\
Pancreas & $\mathbf{3 0}$ \\
& $\mathbf{1 0}$ \\
& $\mathbf{2 0}$ \\
Small bowl & 30 \\
& 10 \\
& $\mathbf{2 0}$ \\
& 30
\end{tabular}

Spleen

775.3

763.81

642.8

659.56

590.1

589.45

975.34

986.98

880.25

$\begin{array}{cc}876.3 & 16.46 \\ 844.74 & 16.37 \\ 684.36 & 16.48\end{array}$

Wavelet Thresholding and Joint NL Means Filtering

16.52

16.1

16.75

16.46

16.48

16.34

16.7

16.81

16.11

16.7

16.4

24.3

24.67

24.31

25.1

25.3

25.11

755.45

23.37

23.47

734.2

23.51 


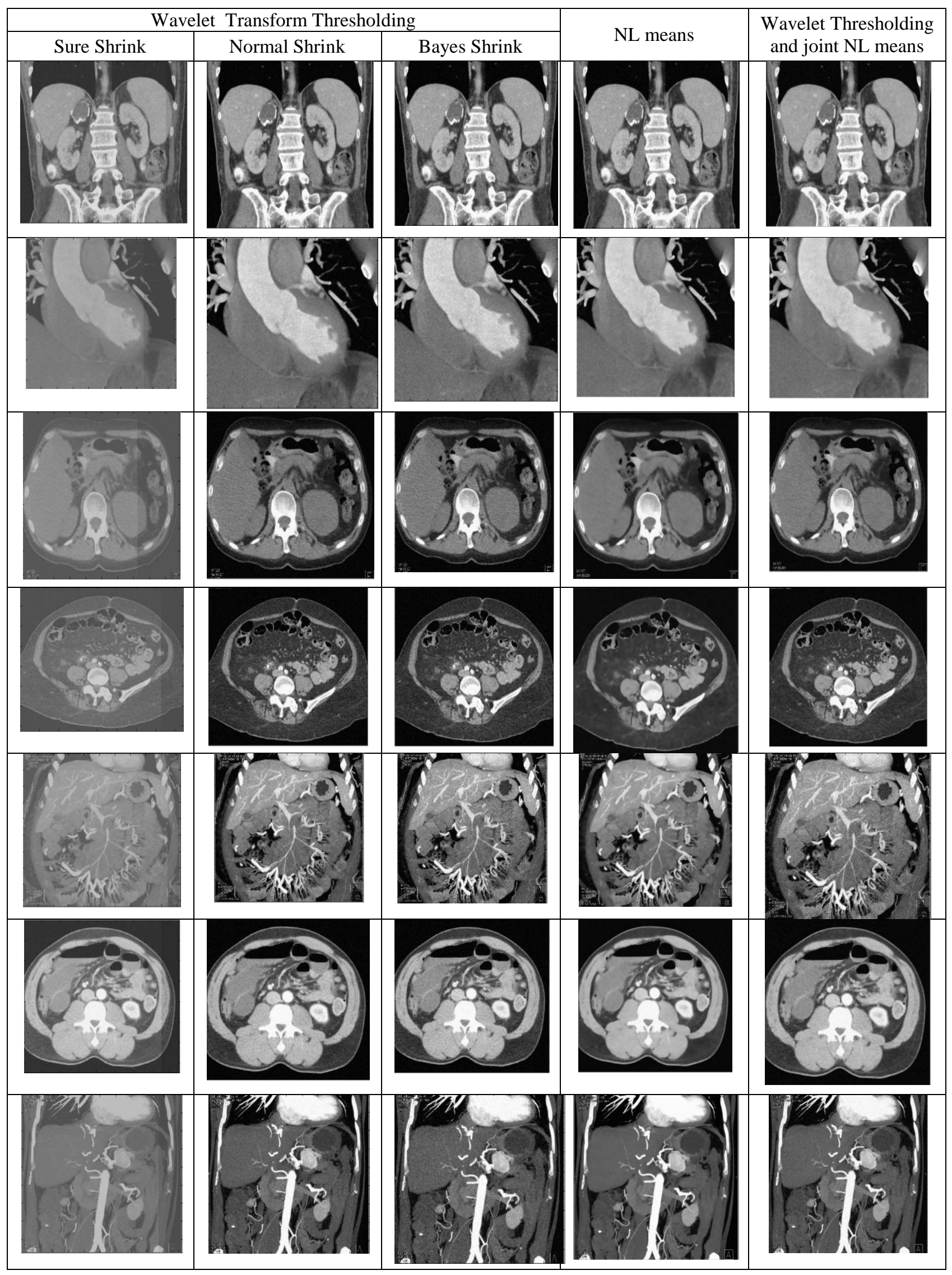

Fig 3 Output Images for noise with std deviation of 20 


\section{IX.CONCLUSION}

CT scan imaging is one of the most widely used imaging modality. The quality of the CT images improves as the radiation dose is increased but exposure to high radiation doses has severe health effects: increasing the risk of life threatening disease like cancer. Low dose images on the other hand have poor quality due to the presence of AWGN noise. Various techniques have been proposed in literature for CT image denoising. This work provides a comparison of three proposed techniques. The wavelet thresholding based denoising results indicate that amongst Visu shrink, normal shrink and Bayes shrink; Bayes shrink outperforms the other two. Although it is computational simple one of the drawbacks observed in this technique is that though it denoises the images it tends smoothens the edges. NL means overcomes this drawback; along with improved denoising it also preserves edges. High computation complexity and computation time are the drawbacks observed in this technique. Using the advantages of both these techniques and also overcoming their drawbacks the third technique that is Wavelet Thresholding and joint NL means based imagedenoising gives the best results. It provides best PSNR and has considerably less computation time compared to NL means.

\section{REFERENCES}

[1] YangWang and Haomin Zhou, "Total Variation Wavelet-Based Medical Image Denoising", International Journal of Biomedical Imaging, Vol. 2006, pp.1-6, January 2006.

[2] G. Beaudoin, and J. A. De Guise, “A method for modelling noise in medical images,” IEEE Trans. Med. Imaging, vol. 23, no. 10,pp. 12211232, Oct. 2004.

[3] T. Lei and W. Sewchand, "Statistical approach to X-ray CT" IEEE Transactions on medical imaging, 1992, pp 53-61

[4] Sudha, Suresh and Sukanesh, "Comparative Study on Speckle Noise Suppression Techniques for Ultrasound Images", International Journal of Engineering and Technology, Vol. 1, No. 1, pp. 57-62, April 2009

[5] www.sprawls.org

[6] A.M.L. Lanzolla, G. Andria, F. Attivissimo, G. Cavone, M. Spadavecchia T. Magli "Denoising Filter to Improve the Quality of CT images", IEEE International Instrumentation and Measurement Technology Conference Singapore, May 2009

[7] S.KotherMohideen Dr. S. ArumugaPerumal, Dr. M.MohamedSathik , “ Image De-noising using Discrete Wavelet transform," IJCSNS International Journal of Computer Science and Network Security, Vol.8 No.1, January 2008.

[8] Savita Gupta, R.C. Chauhan and LakhwinderKaur, "Image denoising using Wavelet Thresholding," Proceedings of the Third Indian Conference on Computer Vision, Graphics Image Processing, Ahmedabad, India, 2002

[9] S.Grace Chang, Bin Yu, Martin Vetterli , “Adaptive Wavelet Thresholding for image denoising and compression,” IEEE Transaction On Image Processing, Vol.9, No.9, September 2000 